\title{
Simultaneous assessment of aberrant retinal vascularization, thickness, and function in an in vivo mouse oxygen-induced retinopathy model
}

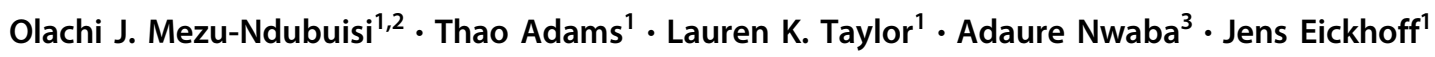

Received: 5 February 2018 / Revised: 26 July 2018 / Accepted: 17 August 2018 / Published online: 12 September 2018

(c) The Royal College of Ophthalmologists 2018

\begin{abstract}
Background Retinopathy of prematurity is a condition of abnormal retinal vascularization in premature infants. The effect of abnormal vascularization on retinal structure and function is unclear. In vivo studies of retinal vascularization, thickness, and function were performed in mice with oxygen-induced retinopathy (OIR mice).

Methods Eighteen mice were exposed to hyperoxia at postnatal day (P) 7, whereas 18 mice were raised in room air (RA). At P20 and 40, electroretinogram was performed for a-wave and b-wave amplitudes and peak times, followed by simultaneous fluorescein angiography for retinal avascular area, arterial tortuosity, and vein dilation assessments, and spectral domain optical coherence tomography for retinal thickness.

Results Capillary density appeared sparser in OIR mice, but retinal avascular area similar to RA mice. Retinal artery tortuosity was higher at P20 and P40 $(P=0.0001)$ in OIR than RA mice. OIR mice had dilated retinal veins at P20 and thinner inner retinas at $\mathrm{P} 40$. Retinal vein width positively correlated with inner retinal thickness $(P=0.008)$. b-wave amplitude was decreased in avascular retinal areas, and correlated with inner retinal thinning. b-wave peak time was prolonged in adult OIR mice at high intensities $(P=0.03)$.

Conclusions Focal variations in retinal vascularization of OIR mice correlate with thickness and function. Adult OIR mice had increased retinal artery tortuosity, prolonged b-wave peak time, and decreased retinal vein width with inner retina attrition. These suggest abnormalities in inner retinal morphology or post-receptor signaling. Studying interactions between retinal vascular, structural, and functional changes could enhance knowledge of OIR pathogenesis and potential therapies.
\end{abstract}

\section{Introduction}

Retinopathy of prematurity (ROP) is a proliferative vascular disease affecting preterm infants exposed to hyperoxia, leading to disorganized vascularization, fibrosis, and potential blindness. ROP is responsible for $6-18 \%$ of

Olachi J. Mezu-Ndubuisi

olachimezu@pediatrics.wisc.edu

1 Departments of Pediatrics, University of Wisconsin School of Medicine and Public Health, 600 Highland Avenue, Madison, WI 53792, USA

2 Ophthalmology and Visual Sciences, University of Wisconsin School of Medicine and Public Health, 600 Highland Avenue, Madison, WI 53792, USA

3 Center for Biotechnology, Krieger School of Arts \& Sciences, Johns Hopkins University, 3400N. Charles St, Baltimore, MD 21218, USA childhood blindness [1]. The most significant risk factors for ROP are oxygen use, prematurity, and low gestational age at birth [2-4], resulting in an incompletely vascularized retina that is vulnerable to the hazards of changing retinal vascular oxygen tension, evidenced by hypoxia-induced increase in arteriovenous difference oxygen tension [5]. ROP is considered to be a vascular disease, but there is mounting evidence of structural abnormalities in human and animal studies [6-8], and functional deficits in children with a history of ROP [8].

Retinal neural, structural, and vascular development are related [9], and so ocular function could be affected by abnormal development of the retinal vessels [10, 11]. Murine models of oxygen-induced retinopathy (OIR) have enhanced the understanding of molecular and cellular aspects of ROP [12, 13]; but it is challenging to examine histological structures without sacrificing the animal. Recent in vivo studies using fluorescein angiography (FA) in live OIR mice have described tortuous retinal arteries, 
widened retinal veins, and reduced capillary density that vary with developmental maturity, with the eventual normalization of retinal vein width, but persistence of tortuosity and reduced capillary density in adult mice [14]. Retinal structural abnormalities have been demonstrated in human ROP [7] and ex vivo animal studies [8]. Spectral domain optical coherence tomography (SD-OCT) is a non-invasive method of studying retinal layer structures using near infrared light to produce fast, high-resolution scans and tomograms $[15,16]$. In an earlier study using SD-OCT in OIR mice after hyperoxia exposure, their retinas were observed to have thinner total cross-sectional width than room air (RA) mice, and avascular retinal areas were shown to be thinner than hypovascular retinal areas [17]. Later in vivo SD-OCT studies revealed that retinal thinning in OIR mice was localized to the inner retina, and was more prominent in the avascular areas [18]. Electroretinogram (ERG) is a commonly used measure of retinal function, but considerable variations in parameters tested (amplitudes, peak times, oscillatory potentials), location tested (focal versus full-field), or flash intensities (low vs medium vs high) make direct comparisons challenging. Whereas noninvasive studies of retinal vascular $[5,14]$ and structural $[17,18]$ development in OIR mice have been shown, simultaneous vascular, structural and functional imaging in the same live animal has not been demonstrated. In this study, we explored whether retinal vascular abnormalities correspond to thickness changes and functional outcomes.

\section{Materials and methods}

\section{OIR}

Newborn C57BL/6 J mice (Jackson Laboratory, Bar Harbor, ME) used for this study were obtained from colonies maintained according to approved protocols by the Institutional Animal Care and Use Committee at University of Wisconsin, Madison, and were in compliance with the Association for Research in Vision and Ophthalmology Statement for the Use of Animals in Ophthalmic and Vision Research. Eighteen mice were exposed to OIR mice by placing them in $77 \pm 2 \%$ oxygen fraction of inspired oxygen in an oxygen chamber (Biospherix ProOX 110; Apex lab, Redwing, MN, USA) with their nursing mothers from postnatal day 7 (P7) to P12, before removal to room air, as previously described in our published modification [5, 14] of an existing mouse model of OIR [19]. Eighteen mice were maintained in room air. At $\mathrm{P} 20$ and $\mathrm{P} 40$, mice underwent ERG followed by simultaneous retinal FA and SD-OCT. The sample size of 18 mice per experimental condition was chosen to detect anticipated large effect sizes of at least 1.0 with $>80 \%$ power at the two-sided 0.05 significance level. Three separate litters of 6-8 pups per litter were used in each age ( $\mathrm{P} 20$ and $\mathrm{P} 40)$ to generate mice used for the study. This study was replicated three times in the laboratory. Animals were randomly allocated to experimental groups. Investigator blinding was not done.

\section{ERG}

The mice were first dark-adapted for $4 \mathrm{~h}$ before ERG was performed using the Micron IV's Labscribe software, according to the manufacturer's specifications. After administering intraperitoneal (IP) ketamine $(100 \mathrm{mg} / \mathrm{kg})$ and xylazine $(10 \mathrm{mg} / \mathrm{kg})$ to induce anesthesia, the mice were then placed on the heated stage of the Micron IV retinal imaging system (Phoenix Research Laboratories) to keep them warm. A paracentral region of interest (ROI) was positioned two disc diameters from the optic nerve in the capillary network between both major vessels (Fig.1a). Flash stimuli were introduced to the retina for a duration of 6 milliseconds ( $\mathrm{ms}$ ) at varying flash intensities to generate a total energy of $0.3 \log \mathrm{cd} \mathrm{sec} / \mathrm{m}^{2}$ (low), $1.8 \mathrm{log} \mathrm{cd} \mathrm{sec} / \mathrm{m}^{2}$ (medium), and $3.3 \mathrm{log} \mathrm{cd} \mathrm{sec} / \mathrm{m}^{2}$ (high). Five recordings were averaged with a $60 \mathrm{~s}$ recovery time between flash intensities. Amplitudes and peak times for a-waves and bwaves were derived. The a-wave is the difference in amplitude between the beginning of the recording and the lowest point or trough of the negative deflection. The bwave amplitude is measured from the lowest deflection or trough of the a-wave to the highest point or peak of the bwave (Fig. 1c). The peak times were determined from the time in milliseconds of light flash initiation to the trough of the a-wave and the peak of the b-wave.

\section{Simultaneous retinal FA and SD-OCT}

After ERG, simultaneous FA and SD-OCT were performed as previously described [18]. The mice pupils were dilated with $1 \%$ tropicamide (Bausch + Lomb, Inc., Tampa, FL), and $10 \%$ sodium fluorescein $(100 \mathrm{mg} / \mathrm{kg})$ (AK-FLUOR, Akorn, Decatur, IL) was injected IP. FA was obtained in all mice (Fig.1a). A circle scan pattern was positioned in a ROI (Fig. 1a), from where ERGs were measured (Fig. 1c), within two disc diameters from the edge of the optic nerve in the vascularized area (RA mice), and in both avascular and hypovascular areas (OIR mice) (Fig. 1c). One circle scan (Fig. 1b) was obtained like previously reported [17]. After image acquisition, semi-automated segmentations using the Micron IV system's Insight software were made to delineate the total, outer, and inner retinal areas [18]. Each SD-OCT image was converted into a retinal thickness color map (Fig. 1b). 
a

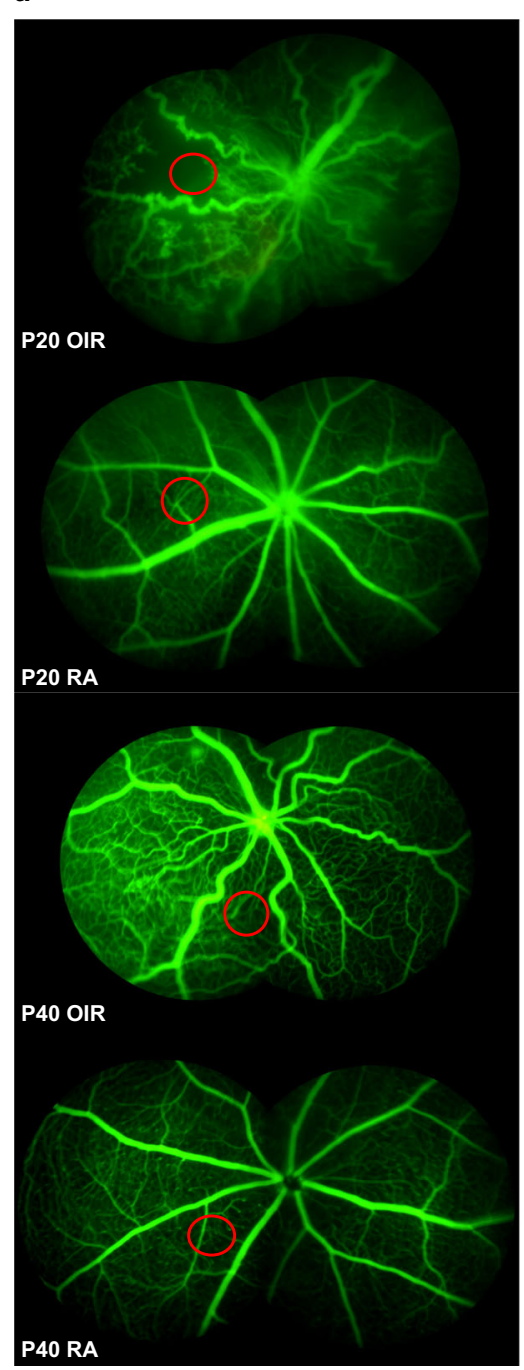

b
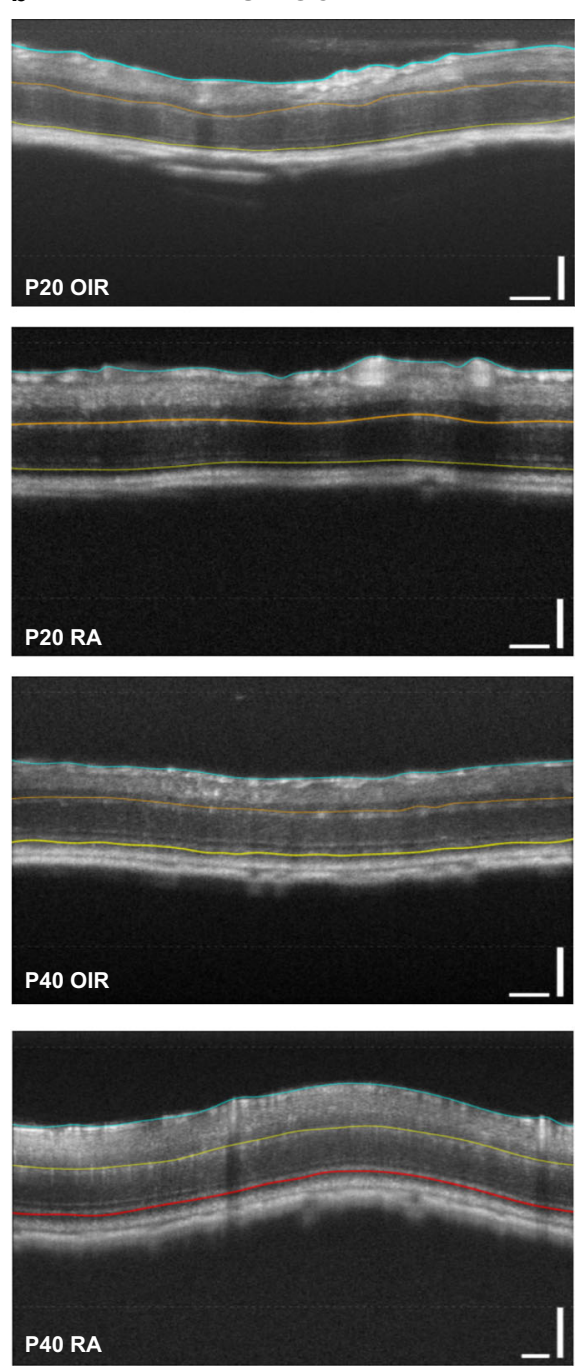

C


Fig. 1 Simultaneous FA, SD-OCT, and ERG. All FA are composite images with the red circle indicating region of interest (ROI) two disc diameters from the optic nerve, where OCT and ERG were measured. The P20 FA image has the following vascular parameter values: RAA $71 \%$, RVW $67.7 \%$, and RAT 1.257. The corresponding SD-OCT image from the same P20 OIR mouse has the following thickness

\section{Quantification of retinal vascular parameters}

For FA, automated and semi-automated customized software developed using MATLAB (Mathworks, Natick, MA), and publicly available at www.quantbv.com, were used to quantify the retinal vascular area (RVA), retinal vein width (RVW), and retinal arterial tortuosity (RAT) in the characterization of the phases of retinal vascular development (RVD), as previously described [14, 18]. For the quantitation of capillary density in this current study, percent retinal avascular area or RAA was used, which is the reverse of percent RVA. RAA is mathematically represented as (100 RVA), and obtained when a retinal FA image, with a centrally values: TRT(vascular) - 166.9, ORT (Vascular): 90.2; IRT Vascular 76.7; TRT-avascular 164.8; ORT (avascular) 89.7, and IRT (Avascular) 75.1. The ERG measured from the same P20 OIR mouse at high intensity $\left(3.3 \log 10 \mathrm{~cd} \mathrm{sec} / \mathrm{m}^{2}\right)$ with a-wave amplitude of $-12 \mu \mathrm{V}$, bwave amplitude of $63 \mu \mathrm{V}$, a-wave peak time of $36 \mathrm{~ms}$, and b-wave peak time of $89.8 \mathrm{~ms}$

located optic nerve, is uploaded in the customized MATLAB software program which automatically converts the FA image to a binary image. An FA image with a peripherally located optic nerve head was used to measure both RVW and RAT using semi-automatic calibrations as previously described [14]. In this study, P20 was selected to represent acute changes in early phase (P16 to P20) RVD, whereas P40 was selected to study chronic changes as there are no differences in vascular and thickness changes between mid (P23 to P27) to late phase (P30 to P34) or mature phases (beyond P35) of RVD (Fig. 1). Mice in each treatment group (RA $n=6$, OIR, $n=8$ ) that developed cataracts during imaging produced blurry images, and were excluded from analysis. 


\section{Statistical analysis}

Data were represented as means and standard deviations. Two-way analysis of variance with post hoc analysis using Tukey's multiple comparisons for pair-wise comparisons were used to determine differences between functional, structural, and vascular parameters at P20 and P40. Bartlett's test was used to verify homogeneity of variances between experimental conditions. The associations between retinal vascular, thickness, and functional parameters were evaluated with Pearson's correlation coefficients and reported with the corresponding $95 \%$ confidence intervals. $P$ values were two-sided and $P<0.05$ was used to define statistical significance. Normal probability and residual plots were examined to verify the normality assumption. Statistical analyses were conducted using SAS software (SAS Institute, Cary NC), version 9.4.

\section{Results}

\section{Comparison of vascular parameters in RA and OIR mice}

The retinas of RA mice were fully vascularized at P20 and P40, whereas the retinas of OIR mice had a patchy vascular phenotype at P20 with avascular areas interspersed with less densely vascularized (hypovascular) areas; and by P40, OIR retinas appeared fully vascularized, but less dense than their age-matched RA counterparts. The retinal avascular area was higher at P20 than P40 in OIR mice $(P=0.0004)$. Contrary to expectation, there was no difference in RAA between OIR and RA mice at P20 $(P=0.42)$ or P40 $(P=$ 0.20). There was no difference in RAA in RA mice at $P 20$ and $\mathrm{P} 40(P=0.08)$. RVW was larger at $\mathrm{P} 20$ in OIR mice than P40 $(P=0.0001)$. P20 OIR mice had larger RVWs than P20 RA mice $(P=0.004)$. There was no difference in RVW between P40 OIR and RA mice $(P=0.45)$. RVW was significantly smaller in P40 than P20 RA mice $(P=$ 0.02) (Fig. 2b, Table 1). OIR mice had more tortuous retinal arteries at $\mathrm{P} 20$ than $\mathrm{P} 40(P=0.002)$. Retinal arteries of OIR mice were more tortuous than those of RA mice at both P20 and $\mathrm{P} 40(P=0.0001)$. There was no difference in RAT between P20 and P40 RA mice. RAA had a weak positive correlation with RVW $(P=0.0486)$ (Fig. 1, Table 1a).

\section{Comparison of SD-OCT parameters in OIR and RA mice}

Inner retinal thickness was significantly reduced in OIR than RA mice at both P20 $(P<0.0001)$ and P40 $(P<$ $0.0001)$. The outer retinal thickness was unchanged in both
OIR and RA mice at both P20 $(P=0.8853)$ and P40 $(P=$ $0.9320)$. In OIR mice, there was a decrease in IRT in vascularized retinal areas with developmental maturity from P20 to P40 in OIR mice $(P<0.0001)$, but no change in ORT from $\mathrm{P} 20$ to $\mathrm{P} 40(P=0.6840)$, resulting in a decrease in TRT from P20 to P40. In RA mice, there was a reduction in IRT from P20 to P40 $(P=0.0001)$, but no change in ORT with developmental maturity from P20 to P40, resulting in a significant decrease in total retinal thickness from P20 to P40 $(P=0.0002)$. The TRT in OIR mice was smaller than RA mice at both P20 $(P<0.0001)$ and P40 $(P<0.0001)$ (Figs. 1, 2, Table 1b). Avascular areas were only noted in the P20 OIR mice. There was no difference in the TRT, ORT or IRT between vascular and avascular areas in the same mouse $(P>0.9)$. In both avascular and vascularized areas of each mouse, IRT was smaller than TRT $(P<0.0001)$, but there was no difference between ORT and IRT $(P=0.09)$.

\section{Comparison of ERG Parameters in OIR and RA mice}

At low intensities, there was no difference in a-wave, bwave, peak time to a-wave, and peak time to b-wave in OIR and RA mice at P20 and P40 $(P>0.05)$. At medium intensities, there was no difference in a-wave, $b$-wave, peak time to a-wave, and peak time to b-wave in OIR and RA mice at $\mathrm{P} 20$ and $\mathrm{P} 40(P>0.05)$. At high intensities, the peak time to $\mathrm{b}$-wave was longer at $\mathrm{P} 40$ in OIR mice compared with RA mice $(P=0.03)$. There was no difference in awave, b-wave, and peak time to a-wave between OIR and RA mice at $\mathrm{P} 40$ at high intensities $(P>0.05)$ (Figs. 1, 3, Table 1c).

\section{Correlation between vascular parameters and retinal thickness}

In hypovascularized and vascularized retinal areas, there was no correlation between percent retinal avascular area and total $(P=0.128)$, inner $(P=0.185)$, or outer $(P=$ $0.057)$ retinal thickness in RA animals, but in OIR mice, percent retinal avascular area had a strong positive correlation with inner retinal thickness $(r=0.68, P<0.001)$ and a marginal positive correlation with total $(P=0.057)$ and outer $(P=0.057)$ retinal thickness. Figure $4 \mathrm{a}$, d, e retinal vein width had a positive correlation with both total $(P=$ $0.003)$ and inner $(P=0.008)$ retinal thickness, but not with outer (P0.0208) retinal thickness in RA mice. In OIR mice, RVW was also positively correlated with total $(P=0.054)$, inner $(\mathrm{P} 0.021)$ retinal, but not outer $(P=0.875)$ retinal thickness. There was no significant correlation between retinal artery tortuosity and any retinal thickness in either RA or OIR mice $(P>0.05)$. 
A

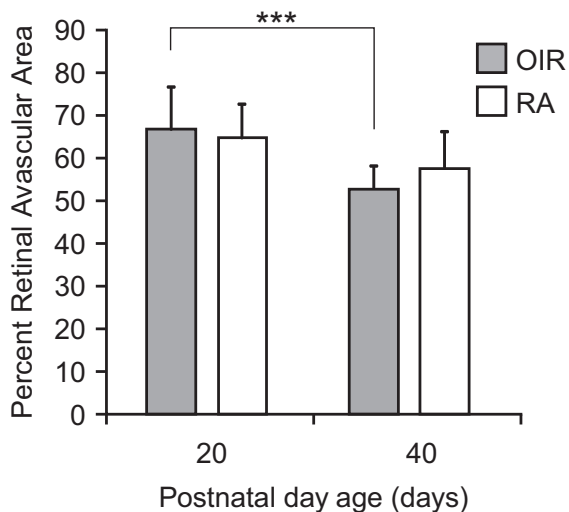

B
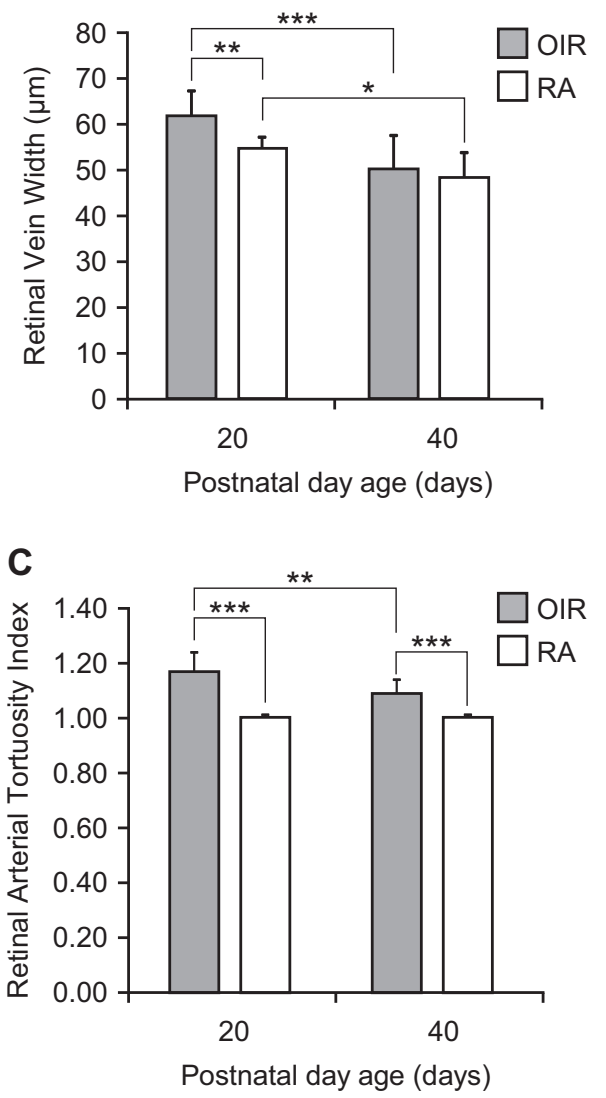

Fig. 2 Vascular and SD-OCT Parameters per Age. Vascular parameters per postnatal day age. a Percent retinal vascular area, b retinal vascular width, c retinal artery tortuosity in oxygen-induced retinopathy (OIR) mice at postnatal day(P) 20 and P40. Retinal thickness parameters per postnatal day age. d Outer retinal thickness, e inner

\section{Correlation between vascular parameters and ERG}

There was no correlation between a-wave, b-wave, and vascular parameters (RAA, RVW, and RAT) $(P>0.05)$ in OIR or RA mice. However, b-wave peak time had a significant positive correlation with RAA $(r=0.57, P=$ $0.015)$, and RVW $(P=0.036)$, but not RAT $(P>0.05)$ in
D

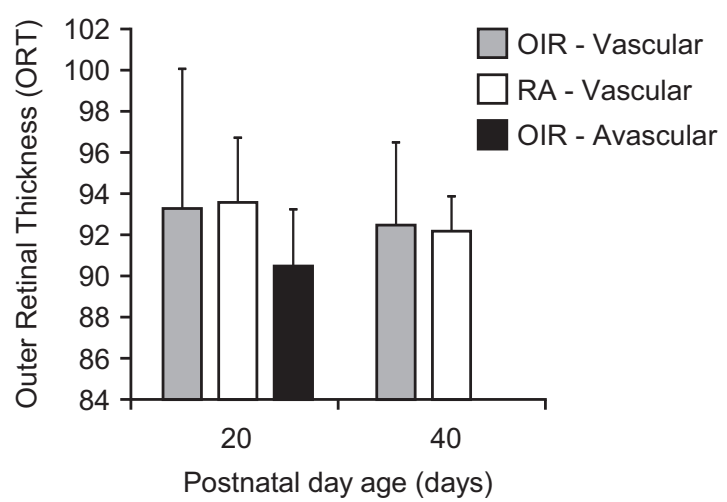

E
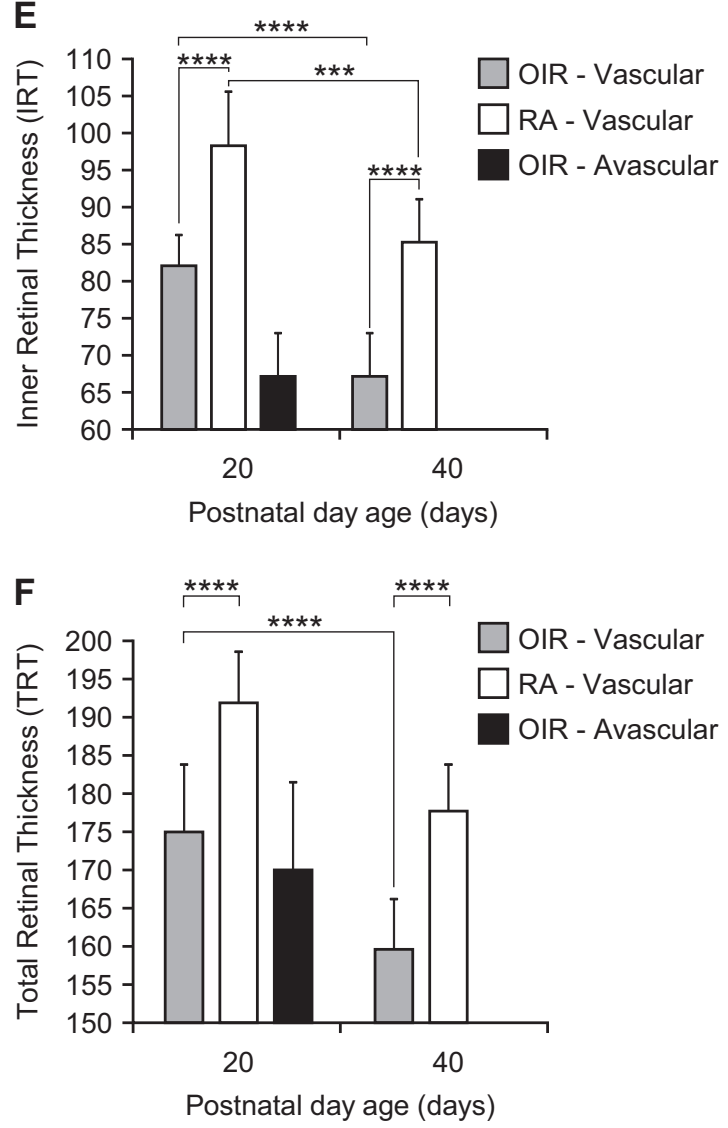

retinal thickness, $\mathbf{f}$ total retinal thickness in OIR and RA mice at P20 and P40. Oxygen-induced retinopathy (OIR); Room Air (RA) 1.1. Vascular parameters per postnatal day age. *Represents a $P$ value of $P<0.05$; **Represents a $P$ value of $P<0.01$; ***Represents a $P$ value of $P<0.001$; ****Represents a $P$ value of $P<0.0001$

RA mice. a-wave peak time had a positive trend in the correlation with RAA in OIR mice $(r=0.43, P=0.07)$, but no correlation was seen in RVW and RAT in OIR mice or RAA, RVW, and RAT in RA mice. b-wave peak time had a trend in the positive correlation with RAA $(r=0.44$, $P=0.07)$ in OIR mice as well, but not with RVW or RAT $(P>0.05)$. 
Table 1 Values for retinal vascular, thickness, and functional parameters in RA and OIR mice

1A. Vascular parameters in OIR and RA mice per age

\begin{tabular}{|c|c|c|c|c|c|c|c|c|}
\hline \multirow{2}{*}{$\begin{array}{l}\text { Age } \\
\text { P20 }\end{array}$} & \multirow[b]{2}{*}{ RAA } & \multicolumn{3}{|c|}{ OIR $($ mean $\pm \mathrm{SD}, n)$} & \multicolumn{3}{|c|}{$\mathrm{RA}($ mean $\pm \mathrm{SD}, n)$} & \multirow{2}{*}{$\begin{array}{l}{ }^{\dagger} P \text { value } \\
0.4229\end{array}$} \\
\hline & & 71.11 & 10.15 & 9 & 67.75 & 8.51 & 8 & \\
\hline & RVW & 62.42 & 5.15 & 9 & 54.49 & 2.46 & 8 & 0.0044 \\
\hline & RAT & 1.16 & 0.07 & 9 & 1.00 & 0.00 & 8 & 0.0001 \\
\hline & ${ }^{\ddagger}$ Weight & 8.44 & 0.73 & 9 & 8.88 & 0.83 & 8 & 0.4600 \\
\hline \multirow[t]{4}{*}{$\mathrm{P} 40$} & RAA & 55.33 & 5.66 & 9 & 60.50 & 9.03 & 10 & 0.1963 \\
\hline & RVW & 50.34 & 7.19 & 9 & 48.48 & 5.19 & 10 & 0.4524 \\
\hline & RAT & 1.09 & 0.05 & 9 & 1.00 & 0.00 & 10 & 0.0001 \\
\hline & $\ddagger$ Weight & 17.44 & 1.81 & 9 & 19.80 & 1.03 & 10 & 0.0001 \\
\hline \multicolumn{9}{|c|}{ 1B. Retinal thickness in RA and OIR mice per age } \\
\hline \multicolumn{2}{|l|}{ Age } & \multicolumn{3}{|c|}{$\mathrm{OIR}($ mean $\pm \mathrm{SD}, n)$} & \multicolumn{3}{|c|}{$\mathrm{RA}($ mean $\pm \mathrm{SD}, n)$} & ${ }^{\dagger} P$ value \\
\hline \multirow{3}{*}{$\begin{array}{l}\text { P20 } \\
\text { vascular }\end{array}$} & TRT & 175.44 & 8.81 & 9 & 191.93 & 6.69 & 8 & $<0.0001$ \\
\hline & ORT & 93.33 & 6.79 & 9 & 93.64 & 3.15 & 8 & 0.8853 \\
\hline & IRT & 82.11 & 4.15 & 9 & 98.28 & 7.32 & 8 & $<0.0001$ \\
\hline \multirow{3}{*}{$\begin{array}{l}\text { P20 } \\
\text { avascular }\end{array}$} & TRT & 170.27 & 11.53 & 9 & $\mathrm{n} / \mathrm{a}$ & & & \\
\hline & ORT & 90.51 & 2.76 & 9 & & & & \\
\hline & IRT & 79.78 & 10.23 & 9 & & & & \\
\hline \multirow{3}{*}{$\begin{array}{l}\text { P40 } \\
\text { vascular }\end{array}$} & TRT & 159.63 & 6.59 & 9 & 177.69 & 6.11 & 10 & $<0.0001$ \\
\hline & ORT & 92.50 & 4.02 & 9 & 92.33 & 1.65 & 10 & 0.9320 \\
\hline & IRT & 67.16 & 5.79 & 9 & 85.34 & 5.80 & 10 & $<0.0001$ \\
\hline \multicolumn{9}{|c|}{ 1C. ERG parameters in RA and OIR per age } \\
\hline \multirow[t]{2}{*}{ Age } & & ERG & \multicolumn{3}{|c|}{ OIR $\left(\right.$ mean \pm SD) $\log 10 \mathrm{~cd} \mathrm{sec} / \mathrm{m}^{2}$} & \multicolumn{3}{|c|}{$\mathrm{RA}($ mean $\pm \mathrm{SD}) \log 10 \mathrm{~cd} \mathrm{sec} / \mathrm{m}^{2}$} \\
\hline & & & 0.3 & 1.8 & 3.3 & 0.3 & 1.8 & 3.3 \\
\hline \multirow[t]{4}{*}{$\mathrm{P} 20$} & & a-wave & $\begin{array}{l}-7.08 \pm \\
6.45\end{array}$ & $\begin{array}{l}-13.34 \pm \\
6.21\end{array}$ & $\begin{array}{l}-29.48 \pm \\
29.05\end{array}$ & $\begin{array}{l}-19.06 \pm \\
22.95\end{array}$ & $\begin{array}{l}-7.40 \pm \\
6.62\end{array}$ & $\begin{array}{l}-22.06 \pm \\
15.82\end{array}$ \\
\hline & & b-wave & $\begin{array}{l}24.76 \pm \\
11.88\end{array}$ & $\begin{array}{l}29.20 \pm \\
7.08\end{array}$ & $\begin{array}{l}67.01 \pm \\
36.13\end{array}$ & $\begin{array}{l}28.83 \pm \\
20.36\end{array}$ & $\begin{array}{l}26.58 \pm \\
11.02\end{array}$ & $\begin{array}{l}75.85 \pm \\
39.38\end{array}$ \\
\hline & & $\begin{array}{l}\text { a-wave } \\
\text { peak } \\
\text { time }\end{array}$ & $\begin{array}{l}45.29 \pm \\
39.84\end{array}$ & $\begin{array}{l}44.58 \pm \\
30.73\end{array}$ & $\begin{array}{l}35.62 \pm \\
13.25\end{array}$ & $\begin{array}{l}60.78 \pm \\
36.25\end{array}$ & $\begin{array}{l}34.93 \pm \\
28.96\end{array}$ & $\begin{array}{l}45.60 \pm \\
36.00\end{array}$ \\
\hline & & $\begin{array}{l}\text { b-wave } \\
\text { peak } \\
\text { time }\end{array}$ & $\begin{array}{l}67.67 \pm \\
45.13\end{array}$ & $\begin{array}{l}80.09 \pm \\
55.18\end{array}$ & $\begin{array}{l}75.87 \pm \\
13.99\end{array}$ & $\begin{array}{l}45.08 \pm \\
36.63\end{array}$ & $\begin{array}{l}78.20 \pm \\
29.45\end{array}$ & $\begin{array}{l}68.50 \pm \\
6.22\end{array}$ \\
\hline \multirow[t]{4}{*}{$\mathrm{P} 40$} & & a-wave & $\begin{array}{l}-22.33 \pm \\
10.30\end{array}$ & $\begin{array}{l}-17.64 \pm \\
10.18\end{array}$ & $\begin{array}{l}-25.48 \pm \\
15.75\end{array}$ & $\begin{array}{l}-22.33 \pm \\
10.30\end{array}$ & $\begin{array}{l}-10.64 \pm \\
9.66\end{array}$ & $\begin{array}{l}-23.66 \pm \\
18.73\end{array}$ \\
\hline & & b-wave & $\begin{array}{l}27.94 \pm \\
16.14\end{array}$ & $\begin{array}{l}25.00 \pm \\
6.38\end{array}$ & $\begin{array}{l}49.68 \pm \\
8.98\end{array}$ & $\begin{array}{l}27.94 \pm \\
16.14\end{array}$ & $\begin{array}{l}33.40 \pm \\
21.99\end{array}$ & $\begin{array}{l}72.45 \pm \\
17.83\end{array}$ \\
\hline & & $\begin{array}{l}\text { a-wave } \\
\text { peak } \\
\text { time }\end{array}$ & $\begin{array}{l}66.33 \pm \\
37.02\end{array}$ & $\begin{array}{l}58.44 \pm \\
37.40\end{array}$ & $\begin{array}{l}58.02 \pm \\
39.20\end{array}$ & $\begin{array}{l}66.33 \pm \\
37.02\end{array}$ & $\begin{array}{l}47.82 \pm \\
40.61\end{array}$ & $\begin{array}{l}71.40 \pm \\
39.45\end{array}$ \\
\hline & & $\begin{array}{l}\text { b-wave } \\
\text { peak } \\
\text { time }\end{array}$ & $\begin{array}{l}53.38 \pm \\
57.30\end{array}$ & $\begin{array}{l}66.82 \pm \\
56.74\end{array}$ & $\begin{array}{l}64.69 \pm \\
7.06^{*}\end{array}$ & $\begin{array}{l}53.38 \pm \\
57.30\end{array}$ & $\begin{array}{l}59.48 \pm \\
40.19\end{array}$ & $\begin{array}{l}57.46 \pm \\
6.00^{*}\end{array}$ \\
\hline
\end{tabular}

P represents postnatal day age; RA represents mice with room air; OIR represents mice with oxygen-induced retinopathy; SD represents standard deviation

${ }^{\dagger} P$ value refers to $P$ value between RA and OIR mice for each postnatal day age P20 or P40

${ }^{\ddagger}$ Weight refers to body weight of each mouse at imaging on P20 or P40, presented in grams

Retinal thickness is presented in $\mu \mathrm{m} ; \mathrm{n} / \mathrm{a}-$ not applicable: there were no avascular areas in RA room air mice at P20, and no avascular capillary areas in both OIR and RA mice at P40

ERG represents electroretinogram values; time to a standards for a-wave peak time; time to $\mathrm{b}$ standards for $\mathrm{b}$-wave peak time. *Represents a $P$ value of $P=0.03$ for b-wave peak. Time at high intensities $\left(3.3 \mathrm{~cd} \mathrm{sec} / \mathrm{m}^{2}\right)$. All other $P$ values for ERG parameters not stated were not significant, $P>0.05$

\section{Discussion}

ROP is more commonly known as a predominantly vascular disease, but there is increasing evidence in clinical and experimental studies that oxidative stress affects the entire retina including its structure and function. In the current study, we found significant spatial and temporal correlations between the acute and long-term effects of hyperoxia on 
Fig. 3 ERG parameters per age. a a-wave amplitude at P20. b awave amplitude at P40. c bwave amplitude at P20.

d b-wave amplitude at P40. e awave peak time at $\mathrm{P} 20$. $\mathbf{f}$ a-wave peak time at $\mathrm{P} 40$. $\mathbf{g}$ b-

wave peak time at $\mathrm{P} 20$. $\mathbf{h}$ b-

wave peak time at P40 and RA mice at P20 and P40
A

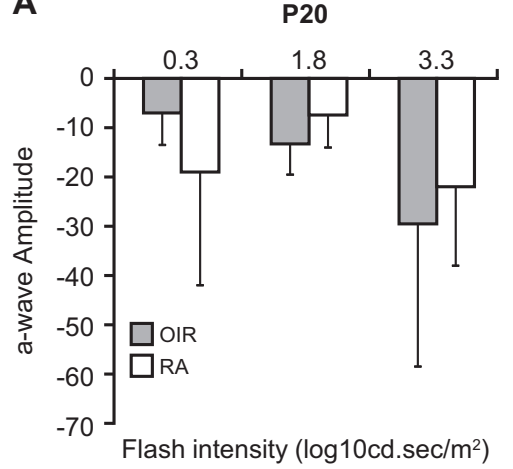

C

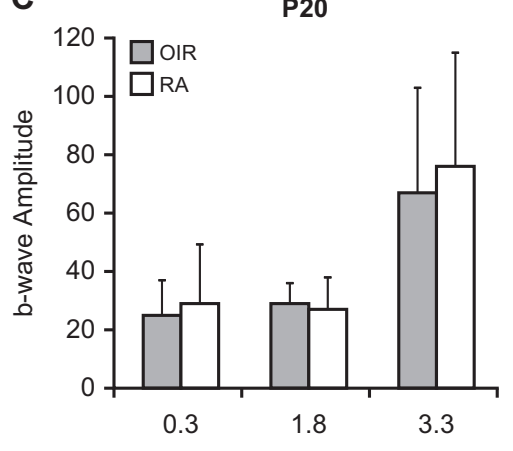

Flash intensity $\left(\log 10 \mathrm{~cd} . \mathrm{sec} / \mathrm{m}^{2}\right)$

E

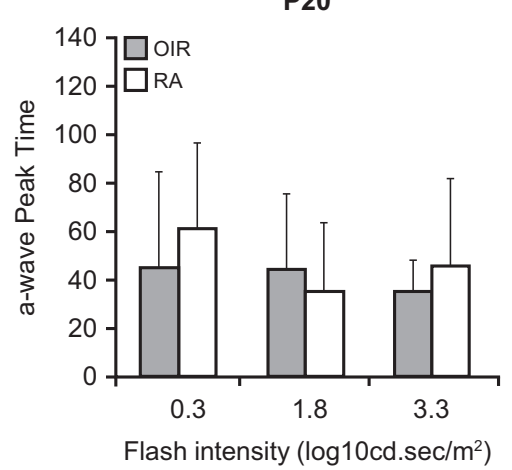

G

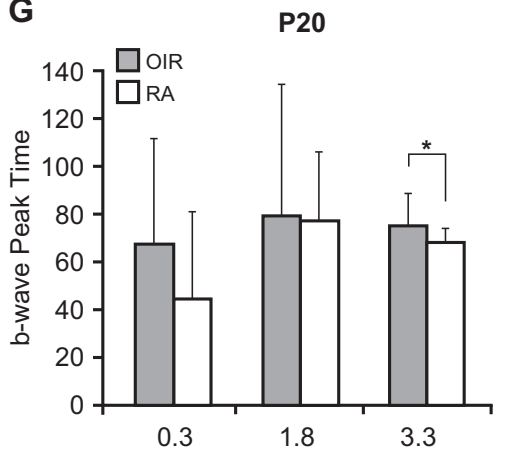

B

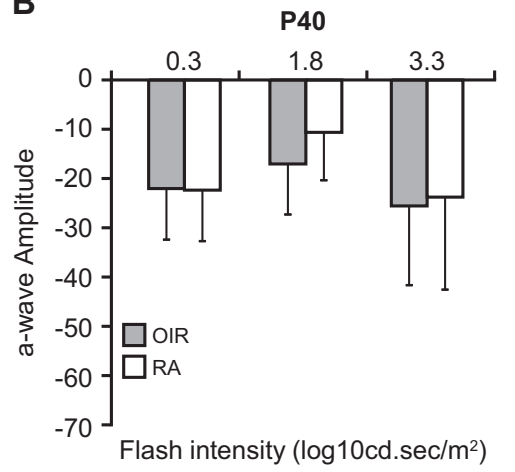

D

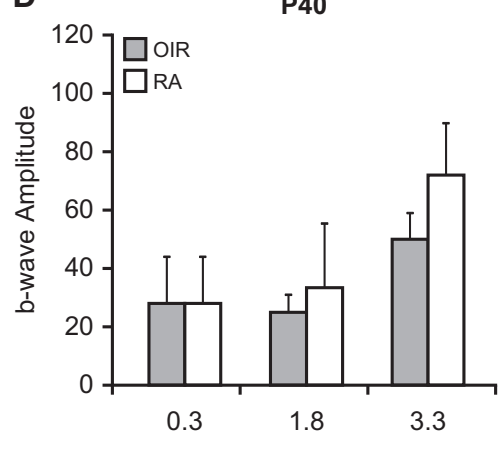

Flash intensity (log10cd.sec/m²)

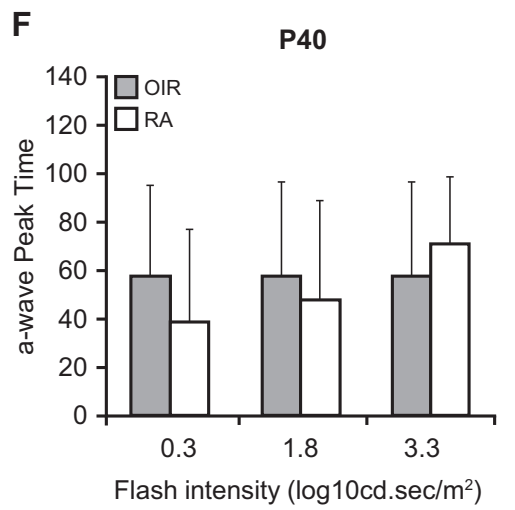

H P40

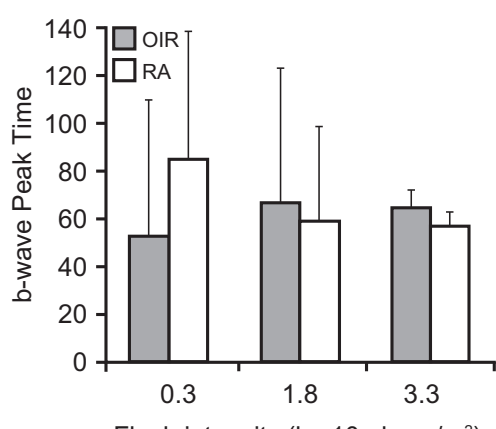

Flash intensity $\left(\log 10 \mathrm{~cd} . \mathrm{sec} / \mathrm{m}^{2}\right)$ retinal vascular development, structure, and function using in vivo techniques in a mouse model of ROP.

Retinal vascular area has been used to quantify the area occupied by retinal veins, arteries, and capillaries in FA images of OIR and RA mice [14]. In this study, retinal avascular area, which is the reverse of RVA, is used. Like earlier studies [14], RA mice had fully vascularized and uniformly dense capillary network, whereas OIR mice 

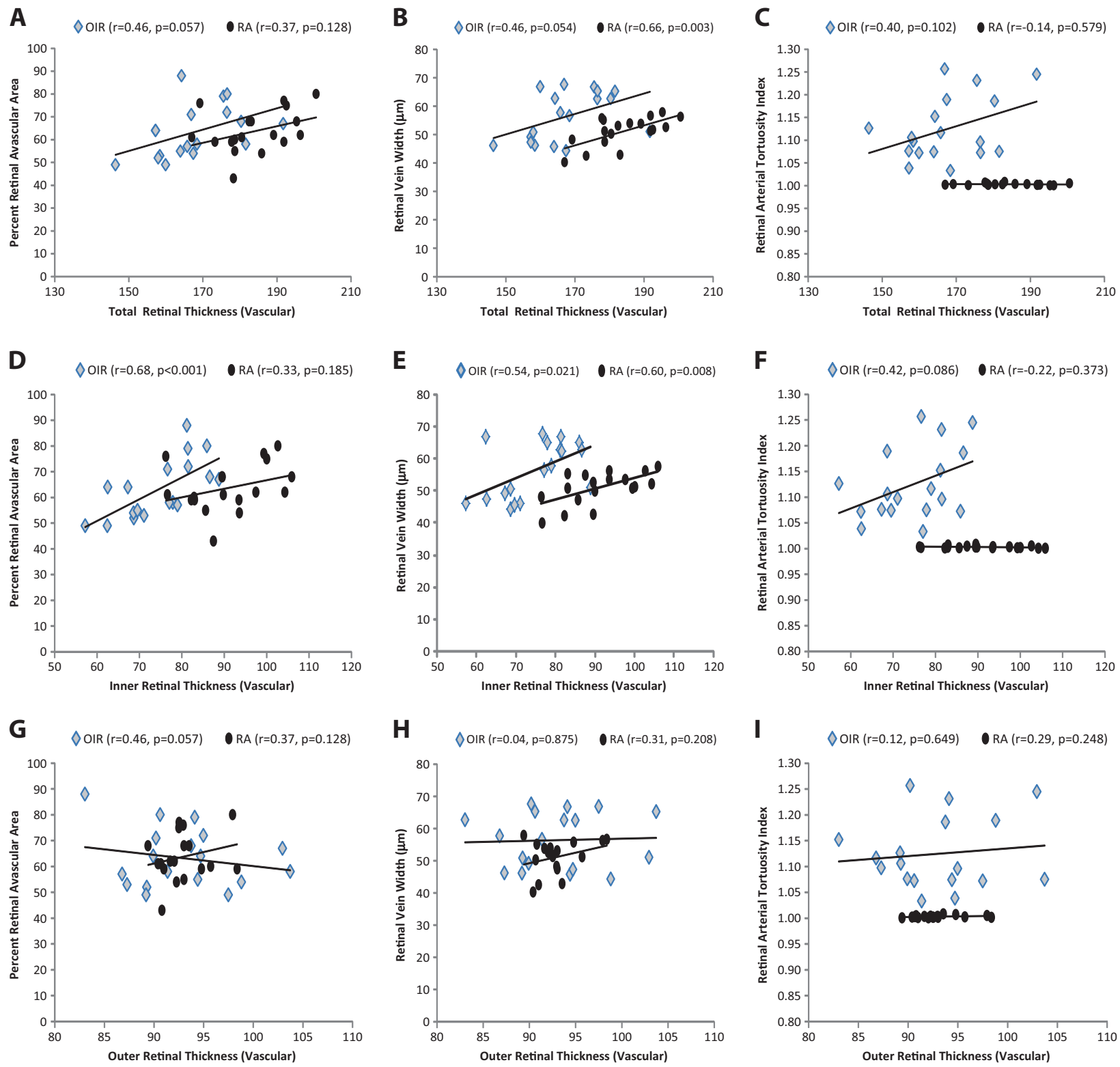

Fig. 4 Correlation of vascular parameters to retinal thickness. Total retinal thickness (TRT) a, b, c inner retinal thickness (IRT) d, e, f outer retinal thickness (ORT) $\mathbf{g}, \mathbf{h}$, i percent retinal avascular area (RAA) a, $\mathbf{d}, \mathbf{g}$ retinal vein width (RVW) $\mathbf{b}, \mathbf{e}, \mathbf{h}$ retinal arterial tortuosity index

showed avascular (no capillary network visible) and hypovascular (less-dense capillary network) retinas. Our current finding of no difference in RVA or RAA between OIR and RA could be due to the larger retinal vein widths at P20 in OIR than RA mice, which would effectively balance out the effect of the avascular phenotype seen in the OIR retina. At $\mathrm{P} 40$, the lack of difference in RAA between RA and OIR mice could be due to the physiologic reduction of vein width during OIR recovery from P20 to P40 to become equivalent to those of RA mice despite persistence of sparse capillary networks in OIR mice. The finding of more tortuous retinal arteries in both neonatal and adult OIR than
(RAT) c, f, i. TRT correlated positively with RAA and RAT. RT correlated positively with RAA and negatively with RAT. There was no correlation between ORT and vascular parameters

RA mice and the decrease in RAA from P20 to P40 in OIR mice (representing an increase in RVA), are both in agreement with previous studies [14]. Retinal veins were larger in OIR mice than RA mice at P20 and similar RVW in mid, late, and mature phases in both RA and OIR mice in agreement with our earlier studies [14]. Neovascularization is a hallmark of human ROP and is quantified in ex vivo OIR studies by measuring areas of hyper fluorescence. In our in vivo mouse model of OIR, whereas signs of 'revascularization' with age are appreciated in the FA images, there is no frank bleeding in the retinas of OIR mice, but rather fluorescein pooling in the vitreous in late 
angiography. Consequently, the consistent phenotype of arterial tortuosity, venous dilation, and vascular-avascular retina are more reliable objective markers for in vivo FA images than "neovascularization", with tortuosity being the most reliable, being higher in neonatal and adult OIR mice compared with age-matched RA mice [14]. RAT, therefore, holds promise as a biomarker for monitoring OIR severity and recovery.

Assessment of retinal thickness is used as a measure of structure in this study. In this current study, we compared inner retinal thickness in avascular areas and vascular areas in OIR mice at P20, as P40 mice had no avascular areas, to find that IRT and ORT were similar in both vascular and avascular areas and significantly smaller than TRT in the same mouse. At P20 and P40, the IRT was significantly lower than TRT in OIR mice, but there were no regional differences between hypovascular and avascular areas in the same OIR mouse. RA mice showed uniform retinal thickness at both P20 and P40, unlike OIR mice. ORT in RA mice was thinner than IRT at P20, but with developmental maturity from P20 to P40, ORT remained stable, whereas IRT decreased in thickness, so that the total retinal thickness was reduced at P40 compared with P20 in RA mice, which is consistent with previous studies [18]. This attrition in inner retinal thickness appears physiologic in RA mice, but is more pronounced in OIR mice. Our findings show that inner retinal thickness was strongly correlated with percent retinal avascular area as well as retinal vein width, indicating that as the area of avascularity decreased or the retina re-vascularized and the dilated retinal veins reduced to normal width in OIR mice, the inner retina became thinner. It appeared that although the retinal veins physically recovered, both the retinal capillaries and inner retinal neuronal cells suffered permanent damage with ongoing attrition of unclear etiology, either owing to cell atrophy or arrested angiogenesis. Programmed cell death, atrophy, or loss from apoptosis have been reported as a cause of retinal thinning in OIR models of ROP and diabetic retinopathy $[13,20,21]$ with retinal atrophy in the INL and the GCL [22, 23], INL and IPL thinning [24], and neuronal apoptosis in avascular retinal regions with localized INL and IPL thinning [25]. Our in vivo studies of retinal structure highlight the vulnerability of the OIR inner retina contributing to long-standing vascular abnormalities. It is unclear if the underlying mechanisms of these signaling abnormalities are due to apoptosis or cell atrophy of inner retinal vascular cells or neuronal cells.

Focal ERG in our study of functional deficits in OIR mice shows prolonged $b$-wave peak time only at high stimuli (3.3 log cd.s $/ \mathrm{m}^{2}$ ), at $\mathrm{P} 40$, but no significant changes in amplitudes or peak times at low or medium flash intensities compared with controls. This could be a result of regional variations owing to the patchy OIR pathology of normal interspersed with abnormal retinal phenotypes; and the focal nature of the testing done may have missed abnormal regions. When grouped correlations between vascular parameters and ERG parameters were evaluated regardless of flash intensity, b-wave peak time was positively correlated with percent retinal avascular area in only RA mice, which suggests that as the RA mice increased in vascular density with age, the b-wave peak time was also decreased. Our finding of prolonged $b$-wave peak times are somewhat consistent with prolonged $b$-wave peak times seen by Lorenc et al.'s ( $130 \mathrm{~ms}$ ) bilateral ERGs at a flash intensity of $5 \mathrm{~cd} \mathrm{~s} / \mathrm{m}^{2}, 0.2 \mathrm{~Hz}$ (roughly $0.7 \log \mathrm{cd} \mathrm{s} / \mathrm{m}^{2}$ ) in overnight dark-adapted P17 OIR mice compared with RA mice with peak times of $50 \mathrm{~ms}$ for both a and b-waves [13]. However, we found this prolonged $b$-wave peak times only in adult mice not neonatal. This variation could be due to the focal variations in the retinal area tested with focal ERG in our monocular study, whereas their study was obtained from bilateral eyes. In contrast to our findings, a full-field mixed (rod and cone) ERG recording in OIR mice found significantly decreasing a-wave and b-wave amplitudes with increasing flashes of light intensities ranging from -3.4 to $1 \log \mathrm{cd} \mathrm{s} / \mathrm{m}^{2}$ compared with RA mice [26], and in another full-field ERG study OIR mice receiving 3-ms flashes at intensities of $0.01-30 \mathrm{~cd} \mathrm{~s} / \mathrm{m}^{2}$ at 1-minute intervals showed reduced a-wave and b-wave amplitudes in addition to prolonged a and $b$-wave peak times in OIR mice. Prolonged b-wave peak time could indicate a delay in the initiation of signal emanating from the inner retina (Muller cells, bipolar cells, amacrine cells, or ganglion cells), either owing to delayed synaptic relay of the electrochemical signal to the inner retinal neurons from the outer retinal neurons (photoreceptor cells) or due to a defect in the structure or function of the inner retinal neurons themselves. Abnormalities in b-wave signaling owing to morphological abnormalities in spatial organization of pre and postreceptor synapses in the ONL or abnormal synaptic processing in the IPL cannot be ruled out. Sparse vascularization in the developing retina could lead to inadequate retinal blood supply with eventual ischemia. Retinal ischemia leads to detrimental molecular cascades resulting in cellular dysfunction and cellular death with subsequent transient or permanent visual impairment [27]. There was only a trend in the positive correlation between RAA and awave or b-wave peak times in OIR mice. a-wave signal from photoreceptors have been reported to appear more resistant to hypoperfusion or hypoxemia unlike the b-wave and oscillatory potentials [28, 29]. Our findings of thinner inner retina and prolonged b-wave peak time suggest abnormal morphology or synaptic interaction in the inner retina, seemingly more severely affected than the outer retina [20,30], or abnormalities in post-receptor signaling in the retina. The role of retinal cellular and neuronal 
involvement in OIR pathogenesis and their functional implications requires further studies.

Limitations of our study include fluorescein leakage from vessels during late angiography, which may affect image quality and lead to overestimation of RVA or underestimation of RAA. Outer retinal abnormalities cannot be ruled out owing to the patchy vascular, structural, and functional phenotype in the OIR retinas. The focal nature of retinal areas tested may have contributed to variability of the vascular, structural, and functional outcomes measured.

In conclusion, oxidative stress transforms the uniformly vascularized, evenly structured, and normally functioning retina into a complex phenotype of patchy focal vascularization, uneven thickness, and function. Total retinal and inner retinal thickness correlated positively with retinal artery tortuosity in neonatal and adult mice indicating the potential utility of tortuosity measurements as a biomarker for OIR recovery. Prolonged b-wave peak times seen in adult OIR mice suggest a delay in the post-receptor responses of the inner retinal cells following signal transmission from the outer retina. Reduction in retinal vein dilation correlated with attrition of the inner retina, and may be a marker for abnormal OIR recovery. Further in vivo and ex vivo OIR studies are needed to localize the specific retinal cellular or neuronal interactions involved during retinal revascularization and their long-term functional implications. This will enable better understanding of ROP pathogenesis and identify novel therapeutic targets.

\section{Summary}

\section{What was known before}

- Retinopathy of prematurity (ROP) is a condition of abnormal retinal vascularization in premature infants. There are limited studies on the effects of oxidative stress on retinal tissue structure and retinal function.

- Retinal neural structural and vascular development are related. The effect of abnormal vascularization on retinal structure and function is unclear.

- Mouse models of oxygen-induced retinopathy are used to study ROP. In vivo techniques would provide more reliable real time information of retinal vascularization, function, and structure, but there are limited in vivo studies in OIR mice.

\section{What this study adds}

- Simultaneous in vivo studies of retinal vessels, structure, and function are possible. Our study shows the feasibility and reproducibility of simultaneous fluorescein angiography to study vascular development, spectral domain optic coherence tomogrpahy to study retinal thickness, and electroretinogram to study retinal function in OIR mice.

- Oxidative stress in the developing retinal affects vessels, tissue structure, and function. Fluorescein angiography shows a patchy vascular-avascular phenotype with increasing capillary vascularization in OIR mice, but persistent capillary sparsity or avascular area, which correlated positively with retinal vein width. SD-OCT shows inner retinal thinning in OIR mice compared with mice raised in room air, and this correlated with retinal artery tortuosity. There was a prolonged b-wave implicit time in adult OIR mice compared with room air.

- Improved understanding of OIR pathogenesis. Inner retinal thinning and prolonged b-wave amplitude suggests atrophy of inner retinal neurons or a delay in the post-receptor responses of the inner retinal cells following signal transmission form the outer retina. Reduction in retinal vein dilation correlated with attrition of the inner retina, and may be a marker for abnormal OIR recovery.

Acknowledgements Funding for this study was provided by the Department of Pediatrics, University of Wisconsin, Madison.

\section{Compliance with ethical standards}

Conflict of interest This authors declare that they have no conflict of interest.

\section{References}

1. Gilbert C. Retinopathy of prematurity: a global perspective of the epidemics, population of babies at risk and implications for control. Early Hum Dev. 2008;84:77-82.

2. VanderVeen DK, Martin CR, Mehendale R, Allred EN, Dammann $\mathrm{O}$, Leviton A, et al. Early nutrition and weight gain in preterm newborns and the risk of retinopathy of prematurity. PLoS ONE. 2013;8:e64325.

3. Hakeem AH, Mohamed GB, Othman MF. Retinopathy of prematurity: a study of prevalence and risk factors. Middle East Afr J Ophthalmol. 2012;19:289-94.

4. Brown BA, Thach AB, Song JC, Marx JL, Kwun RC, Frambach DA. Retinopathy of prematurity: evaluation of risk factors. Int Ophthalmol. 1998;22:279-83.

5. Mezu-Ndubuisi OJ, Teng PY, Wanek J, Blair NP, Chau FY, Reddy NM, et al. In vivo retinal vascular oxygen tension imaging and fluorescein angiography in the mouse model of oxygeninduced retinopathy. Invest Ophthalmol Vis Sci. 2013;54: 6968-72.

6. Ecsedy M, Szamosi A, Karko C, Zubovics L, Varsanyi B, Nemeth $\mathrm{J}$, et al. A comparison of macular structure imaged by optical coherence tomography in preterm and full-term children. Invest Ophthalmol Vis Sci. 2007;48:5207-11. 
7. Maldonado RS, O'Connell R, Ascher SB, Sarin N, Freedman SF, Wallace DK, et al. Spectral-domain optical coherence tomographic assessment of severity of cystoid macular edema in retinopathy of prematurity. Arch Ophthalmol. 2012;130:569-78.

8. Nakamura S, Imai S, Ogishima H, Tsuruma K, Shimazawa M, Hara $\mathrm{H}$. Morphological and functional changes in the retina after chronic oxygen-induced retinopathy. PLoS ONE. 2012; $7: \mathrm{e} 32167$.

9. Fulton AB, Hansen RM, Moskowitz A, Akula JD. The neurovascular retina in retinopathy of prematurity. Prog Retin Eye Res. 2009;28:452-82.

10. Mowat FM, Gonzalez F, Luhmann UF, Lange CA, Duran Y, Smith AJ, et al. Endogenous erythropoietin protects neuroretinal function in ischemic retinopathy. Am J Pathol. 2012;180: 1726-39.

11. Hansen RM, Moskowitz A, Akula JD, Fulton AB. The neural retina in retinopathy of prematurity. Prog Retin Eye Res. 2017;56:32-57.

12. Ishida S, Usui T, Yamashiro K, Kaji Y, Amano S, Ogura Y, et al. VEGF164-mediated inflammation is required for pathological, but not physiological, ischemia-induced retinal neovascularization. $\mathrm{J}$ Exp Med. 2003;198:483-9.

13. Lorenc VE, Caldarone PVS, Paz MC, Ferrer DG, Luna JD, Chiabrando GA, et al. IGF-1R regulates the extracellular level of active MMP-2, pathological neovascularization, and functionality in retinas of OIR mouse model. Mol Neurobiol 2018;55: 1123-35.

14. Mezu-Ndubuisi OJ. In vivo angiography quantifies oxygeninduced retinopathy vascular recovery. Optom Vis Sci. 2016; 93:1268.

15. Gordon-Lipkin E, Calabresi PA. Optical coherence tomography: a quantitative tool to measure neurodegeneration and facilitate testing of novel treatments for tissue protection in multiple sclerosis. J Neuroimmunol. 2017;304:93-6.

16. Calabresi PA, Balcer LJ, Frohman EM. Optical coherence tomography in neurologic diseases: Cambridge University Press; 2015.

17. Mezu-Ndubuisi OJ, Wanek J, Chau FY, Teng PY, Blair NP, Reddy NM, et al. Correspondence of retinal thinning and vasculopathy in mice with oxygen-induced retinopathy. Exp Eye Res. 2014;122:119-22.

18. Mezu-Ndubuisi OJ, Taylor LK, Schoephoerster JA. Simultaneous fluorescein angiography and spectral domain optical coherence tomography correlate retinal thickness changes to vascular abnormalities in an in vivo mouse model of retinopathy of prematurity. J Ophthalmol. 2017;2017:9620876.

19. Smith LE, Wesolowski E, McLellan A, Kostyk SK, D'Amato R, Sullivan R, et al. Oxygen-induced retinopathy in the mouse. Invest Ophthalmol Vis Sci. 1994;35:101-11.

20. Dorfman AL, Cuenca N, Pinilla I, Chemtob S, Lachapelle P. Immunohistochemical evidence of synaptic retraction, cytoarchitectural remodeling, and cell death in the inner retina of the rat model of oygen-induced retinopathy (OIR). Invest Ophthalmol Vis Sci. 2011;52:1693-708.

21. Yamaguchi $M$, Nakao $S$, Arita $R$, Kaizu $Y$, Arima $M$, Zhou Y, et al. Vascular normalization by ROCK inhibitor: therapeutic potential of ripasudil (K-115) eye drop in retinal angiogenesis and hypoxia. Invest Ophthalmol Vis Sci. 2016;57: 2264-76.

22. WOLTER JR. Diabetic capillary microaneurysms of the retina. Arch Ophthalmol. 1961;65:847-54.

23. Bek T. Transretinal histopathological changes in capillary-free areas of diabetic retinopathy. Acta Ophthalmol (Copenh). 1994;72:409-15.

24. Barber AJ, Lieth E, Khin SA, Antonetti DA, Buchanan AG, Gardner TW. Neural apoptosis in the retina during experimental and human diabetes. Early onset and effect ofinsulin. J Clin Investig. 1998;102:783.

25. Sennlaub F, Courtois Y, Goureau O. Inducible nitric oxide synthase mediates retinal apoptosis in ischemic proliferative retinopathy. J Neurosci. 2002;22:3987-93.

26. Martini D, Monte MD, Ristori C, Cupisti E, Mei S, Fiorini P, et al. Antiangiogenic effects of $\beta 2$-adrenergic receptor blockade in a mouse model of oxygen-induced retinopathy. $J$ Neurochem. 2011;119:1317-29.

27. Yang D, So KF, Lo AC. Lycium barbarum polysaccharide extracts preserve retinal function and attenuate inner retinal neuronal damage in a mouse model of transient retinal ischaemia. Clin Exp Ophthalmol. 2017;45:717-29.

28. Block F, Schwarz M. The b-wave of the electroretinogram as an index of retinal ischemia. Gen Pharmacol. 1998;30:281-7.

29. Kang Derwent JJ, Linsenmeier RA. Hypoglycemia increases the sensitivity of the cat electroretinogram to hypoxemia. Vis Neurosci. 2001;18:983-93.

30. Cheng H, Nair G, Walker TA, Kim MK, Pardue MT, Thulé PM, et al. Structural and functional MRI reveals multiple retinal layers. Proc Natl Acad Sci. 2006;103:17525-30. 